\title{
Mathematics: Its content, methods, and meaning
}

\section{by Shing-Tung Yau*}

In this article, we will explore the contents, methods, and meaning of mathematics. I have borrowed the title from a book compiled by three members of the former Soviet Union's mathematics community: A. D. Aleksandrov, A. N. Kolmogorov, and M. A. Lavrent'ev.

In 1998, Peking University celebrated the 100th anniversary of its establishment, and it was on this occasion that I decided to tackle this important and unendingly interesting subject. ${ }^{1}$ As such, it seems fitting to set the proverbial stage with a riotous event centered at this university: the May Fourth Movement of 1919. This was a student movement in Beijing that arose in protest to the way in which the Chinese government responded to the Treaty of Versailles, which marked the resolution of World War I. The importance of the May Fourth movement is found within the fact that this movement culminated in the march of over 3,000 Peking University students, as well as students from other neighboring schools, on Tiananmen to express their discontent over the way the situation was handled and to voice their desire for modernization taken by their Western counterparts.

The significance of this tumultuous event to our story is that it highlights the dispute between Chinese and Western culture, which was the central player in the events surrounding the May Fourth Movement. I will use this dispute to clarify certain aspects of mathematics in China and their meaning, and so we will briefly recount the history behind the conflict here. During the mid-nineteenth century, China's understanding of Western science and technology es-

\footnotetext{
* Department of Mathematics, Harvard University

E-mail:yau@math.harvard.edu

${ }^{1}$ A portion of the contents of this paper have been adapted from a speech given by the author at the 100th anniversary ceremony for Peking University in 1998. I thank Yong Lin, Jason, Hung Hsi Wu for helping with translation of this article.
}

sentially boiled down to "powerful ships and armaments." After losing wars again and again, Zhidong Zhang, a prominent official in the Chinese government during the latter years of the Qing Dynasty, proposed a program of "Western learning for practical applications and Chinese learning providing the fundamental structure." Namely, to add Western technology for practical purpose, but to retain the traditional Confucianist spirit as the basis of society. It was at this moment that the debate between science and metaphysics was awoken within China.

A faction of the society led by Shuming Liang, a notable educator and philosopher in the late Qing Dynasty, wanted a return to the Eastern tradition of spirituality and humanism, with Confucianism at its core. They saw Western civilization's emphasis on rationalism and scientific knowledge in order to achieve mastery over the natural world, as a departure from the essence of human existence. They envisioned that it would lead to the enslaving of humanity by machines. Chinese culture in their view, however, is both self-adaptive and self-sufficient, which allows it to take a sort of middle ground upon which it can firmly progress forward. This view was, in some sense, echoed by the views of the illustrious Western philosopher Bertrand Russell toward the end of World War I, who felt a tremendous abhorrence for the growing materialistic nature of Western civilization, and who advocated for learning from the East. This view was not, however, universally held within China; the nation found itself embroiled in a tense debate over which way the country should proceed. A noteworthy example of the opposition to the views held by Liang and his followers is found within a faction headed by Shi $\mathrm{Hu}$, who argued that science is capable of achieving anything within the realm of knowledge. Moreover, Hu's followers felt that even philosophy is subservient to the scientific 
method, and that philosophical views that have not been closely critiqued by logical reasoning cannot claim to constitute genuine knowledge.

Ultimately, no final resolution of this raging conflict between science and metaphysics has been reached to this day. One of the primary reasons for lack of a conclusion was that neither of the opposing factions devoted itself to any serious study of the basics of modern science and that most of them do not appreciate the fact that theoretical speculations must be supported by a robust logical framework and hard experimental data. Ultimately, the advocacies of both sides degenerated into nothing more than empty rhetoric. This, in fact, epitomizes one of the defining characteristics of traditional Chinese culture. On the one hand, some of the Chinese philosophical points of view are extremely abstract and sometimes without substance. Indeed, such abstraction is markedly present in Confucianism and Taoism, both of which preach harmony between human beings and nature, as well as in Zen Buddhism, which insists on freeing oneself from the written word and focusing on one's soul. On the other hand, traditional Chinese philosophy can be extremely practical. Chuang Tzu, an immensely influential philosopher of the early Taoist movement, said, "People are always blinded by happiness but don't know the reality of the world." This quote suggests that the pursuit of the intangible (impractical) can cause one to miss the reality in front of them (practical). Such attention to practicality pervades much of ancient Chinese science, as exemplified by the fact that the four great inventions of ancient China-the compass, paper-making, printing, and gunpowder-were each used to serve the practical needs of people. It is in this latter characteristic that traditional Chinese ideals find essential difference from those of the West, which are based on the development of basic science. I believe that basic science provides a bridge between reality and the abstract, as well as providing us with an essential tool for understanding the universe surrounding us; and the language of basic science is mathematics.

Many scientists throughout history have highly praised mathematics - this is evident in the words of numerous celebrated physicists. One such example can be found in the words of American physicist R. P. Feynman, who said in his book The Character of Physical Law that "Every one of our laws is a purely mathematical statement in rather complex and abstruse mathematics... Why? I have not the slightest idea." Another prime example comes from the Hungarian American theoretical physicist E. Wigner's classic essay The Unreasonable Effectiveness of Mathematics in the Natural Sciences, in which he concluded: "The miracle of the appropriateness of the language of mathematics for the formulation of the laws of physics is a wonderful gift which we neither understand nor deserve. We should be grateful for it and hope that it will remain valid in future research and that it will extend, for better or for worse, to our pleasure, even though perhaps also to our bafflement, to wide branches of learning." Still another variation on this theme comes from the British-American theoretical physicist F. Dyson, who said, "One factor that has remained constant through all the twists and turns of the history of physical science is the decisive importance of the mathematical imagination." Even outside of the physics community this was recognized by, for example, H. D. Thoreau, a nineteenth-century American philosopher, who wrote in his seminal work Walden that "The most distinct and beautiful statement of any truth must take at last the mathematical form."

In fact, the art of mathematics not only absorbs vital nutrients from nature, but also derives enormous inspiration from the both the social and engineering sciences. Upon receiving inspiration from any variety of phenomena, the human soul discerns the outline of beauty; and the elements contained within this domain which can be handled by strict adherence to the laws of logic are what mathematicians study. The difference between mathematics and other sciences lies in the fact that mathematics incorporates the abstract. Some mathematicians believe that, as long as a theorem is beautiful, it has the power to resolve questions of importance in nature. Aside from the great influence afforded aesthetics, another key difference between mathematics and, say, literature is that all propositions can be deduced from a comparatively small collection of easily recognized axioms. The origins of this aspect of mathematics, as a systematic science, can be traced back to the prodigious geometer Euclid, whose Elements stood as an imperishable masterpiece in ancient Greece. Upon translating this jewel of mathematics into Chinese, the Italian Jesuit priest Matteo Ricci and the Chinese scholar and scientist Guangqi $\mathrm{Xu}$ remarked: "There are more than five hundred questions in 13 volumes; there are interconnections between the volumes and between the propositions. Their sequencing is rigid and cannot be perturbed with impunity, and in their step-by-step build-up to the inevitable conclusions, one finally perceives the profound truths." Theorems, no matter how deep or subtle, can nevertheless be deduced from a few simple axioms. Beauty is finally united with truth, and they can no longer be separated. It is worth pointing out that Euclid's understanding of mathematics directly influenced the thoughts of Newton and his famous three laws of motion. Indeed, the style of writing of Newton's magnum opus, Philosophice Naturalis Principia Mathematica (The Mathematical Principles 
of Natural Philosophy) grows directly out of the axiomatic structure of Euclid's Elements. Euclid's influence extends far beyond Newton. The recent quest for Unified Field Theory-the dream of all modern physicists starting with Einstein-is nothing but an expression of the same desire to explain all the forces in the universe from a few basic principles.

Mathematicians have a deep understanding of the harmony between truth and beauty. For example, English mathematician J. J. Sylvester said: "The world of ideas which [mathematics] discloses or illuminates, the contemplation of divine beauty and order which it induces, the harmonious connexion of its parts, the infinite hierarchy and absolute evidence of the truths with which it is concerned, these, and such like, are the surest grounds of the title of mathematics to human regard, and would remain unimpeached and unimpaired were the plan of the universe unrolled like a map at our feet, and the mind of man qualified to take in the whole scheme of creation at a glance." Echoing this, the historian of mathematics M. Kline said, "An elegantly executed proof is a poem in all but the form in which it is written."

In fact, having absorbed the essence of the natural sciences, mathematicians then yield control to their imagination-strictly counseled by aesthetic aspirations and the restrictions of logic-to ultimately create genuinely amazing propositions which astonish even the mathematicians themselves. As a prime example of this occurrence, consider the resolution of the Weil Conjecture, which led to a complete restructuring of arithmetic geometry, and which blended topological and algebraic geometry into the theory of equations of integers. A. Grothendieck and P. Deligne's proof of the Weil Conjecture is rightly viewed as a colossal achievement in the grand program of releasing oneself to the realm of abstract ideas.

When recounting the history of mathematics, we find ourselves encountering a plethora of advances that were obtained via the natural integration of several diverse and salient concepts. Such occurrences have become the great milestones of mathematical development. Einstein famously merged the seemingly disparate concepts of time and space, an accomplishment which is universally recognized as a cornerstone of twentieth-century physics. Another soulstirring example is A. Wiles study of automorphic forms and their relation to Fermat's Last Theorem. It is remarkable that mathematicians are capable of achieving such towering feats without depending on inspiration from the natural sciences. The reason for this capability can be traced back to the fact that both math and the notion of space are themselves part of nature, and their immense structure constitutes an integral part of the structure of the universe. We should keep in mind, however, that the mystery of nature is too deep to be fathomed, and its perfection is not only found in math and the concept of space, but everywhere; it is for this reason, therefore, that mathematicians cannot and should not resist the pull of nature.

The two major discoveries in physics of the twentieth century-relativity and quantum mechanics-had a deep impact on mathematics. General relativity makes differential geometry "having substance in nature", and Riemannian geometry is no longer merely an abstract branch of mathematics. Beyond this, from the beginning, quantum field theory cast mathematicians into a state of chaos, for its foundation is not clear. However, its effect on mathematics has been like magic. The application of the Dirac equation to geometry, for example, is quite elusive, and forcefully affected the development of geometry. More recently, supersymmetry-a concept developed by physicists within the past forty years - stands as another bizarre concept, in both experimental and theoretical terms. Through the help of superstring theory, however, mathematicians have developed techniques for solving numerous difficult mathematical problems that have remained open for centuries. The utility and importance of superstring theory in mathematics is unquestionable, and so, unless the gods of destiny wish to make fools of humanity, it will ultimately find its place in physics as well.

At the end of the nineteenth century, the axiomatization movement laid a solid foundation for mathematics. Mathematicians were under the impression that they had the necessary tools to resolutely move forward with no more fear about issues of rigor. Indeed, at the beginning of the twentieth century Hilbert thought that all propositions in the realm of mathematics could be deduced from a complete set of simple axioms. However, in his famous 1931 paper Über formal unentscheidbare Sätze der Principia Mathematica und verwandter Systeme, I (On Formally Undecidable Propositions of Principia Mathematica and Related Systems, I), Gödel proved that any axiomatic system sufficiently robust to incorporate standard logic and elementary number theory cannot be established. This means that Hilbert's idea is not completely sound and science is not omnipotent. Nevertheless, insofar as the problems that arise from the natural world are concerned, we believe that Hilbert's ideas are basically correct.

Having obtained some understanding of the diversity of mathematics, I now turn to the question: What is it, exactly, that mathematicians do? To help answer this, I will roughly classify mathematicians into three types, based on their intrinsic features, as seen below. 


\section{Mathematicians Who Produce Theory}

The working patterns of a mathematician belonging to this class can be further subdivided into essentially seven categories.

a. The first category includes mathematicians who catch glimpses of far-reaching commonalities between phenomena, and who, from this, refine the behavior relevant to what they wish to study into a set of basic principles. These principles can then be employed to explain a number of similar issues in a systematic manner. An obvious example of this manner is S. Lie, who, after observing numerous symmetrical phenomena in both mathematics and physics at the end of the last century, created the theory of continuous group transformations in relation to differential equations. The concept of a Lie group has become a crucial and basic concept in modern mathematics.

b. Another category of mathematicians extend and graft existing theoretical constructs onto other structures. Examples of this include: calculus being extended from finite dimensional spaces to infinite dimensional spaces, calculus being utilized on surfaces in order to obtain connection theory, and so forth. Indeed, when Ricci, Christoffel, and various other geometers began researching connection theory on surfaces (without dependence on the choice of coordinates), it was difficult for them to imagine that it would become so important to Yang-Mills field theory decades later.

c. The pursuit of commonality between different subjects naturally lends itself to the discovery of new results via some manner of comparison method. For example, A. Weil developed arithmetic geometry through comparisons between equations of integers and algebraic geometry. Another important illustration of this paradigm is found in R. Langlands, who was led to proposing the so-called Langlands program, by seeking to combine the representation theory of groups and automorphic forms, which extended commutative class field theory to noncommutative class fields.

d. New theoretical developments arise out of the attempt to explain new phenomena uncovered in mathematics. An example of this theme comes after Gauss' discovery that the curvature of a surface is intrinsic (i.e. it is only relevant to its first fundamental form), Riemann subsequently created a major field in geometry that now bears his name, and this new kind of geometry has been essential in the developments in geometry within the recent centuries. After $\mathrm{H}$. Whitney unmasked new invariants of a fiber bundle that he called "characteristic classes." L. Pontryagin and S. S. Chern generalized his idea to more gen- eral cases. Today, Chern classes have become some of the most basic invariants.

e. Much theoretical development is fueled by the desire to solve important issues currently plaguing the mathematical community. For instance, in order to solve the problem of embedding a general Riemannian manifold into Euclidean space isometrically, J. Nash developed the implicit function theorem and this led to the formation of an entirely new subject, which proved incredibly useful in studying differential equations. Another example is seen in S. Smale's solution of the Poincaré conjecture in dimension at least five by employing h-cobordism theory, this theoretical construct became one of the most important tools for differential topology.

f. The proofs of new theorems often point to the need for deeper theoretical developments. Thus the Atiyah-Singer index theorem and Donaldson's theory were followed by many different proofs which in turn led to much important work.

g. Lastly, mathematicians of this category often confer a new structure that leads to promising new areas of research. Example include: Kähler, after introducing his eponymous metric when researching complex manifolds; Thurston's recent introduction of the concept of geometrization when studying threedimensional manifolds; and so on. In general, the introduction of a new structure gives a meaningful research direction. However, one often needs to impose restrictions on the structure, e.g., construction of Ricci flat metrics on Kähler manifolds with zero first Chern Class.

\section{Mathematicians Seeking Laws Underlying Natural Phenomena}

This class of mathematician works on data, experiments, explores problems deemed worthy of research in light of their connection to natural and social phenomena, distills the key ideas, and makes significant conjectures. For example, Gauss proposed the form of the asymptotic distribution of prime numbers amongst the integers after inspecting a large collection of prime numbers himself. Another example would be the letters between B. Pascal and $P$. Fermat regarding the odds in gambling, which laid the foundation of modern probability theory. F. Black and M. Scholes proposed the equation describing option pricing, once the futures market had emerged in 1950s, and this was immediately and widely applied in options dealing. Scholes won the Nobel Prize in Economics in 1997 for this influential work. There are, indeed, too many examples of this nature to enumerate.

In any case, it is not easy to make good conjectures in mathematics. One has to have an adequate 
understanding of the given situation before one can do that. A good example appears in Chinese literature: It is possible to form a good idea of the final outcome of the famous Chinese novel, The Dream of the Red Chamber, by reading the first sixty or seventy chapters. However, one cannot do that if one does not understand the poetry or the imageries in those sixty or seventy chapters.

\section{Mathematicians Who Solve Difficult Problems}

All mathematical theories must ultimately lead to the solution of some important problems-otherwise this theory would be empty and valueless. The significance of a new theoretical framework must be proportional to that of the problems it has the ability to solve. Indeed, the significance of a difficult mathematical problem lies in whether or not it produces an abundant new theoretical world in which we can explore. A single, wonderful proof is not the only part of mathematics. To understand this point a little more clearly, consider the example of the four-color problem-it cannot be said that a large amount of significant mathematics has resulted from its solution almost forty years ago.

On the other hand, there have been countless unyieldingly formidable problems whose resolution moved the relevant topics into the mainstay; only by overcoming them can we reach a deep understanding of the subject. For instance, we cannot say that we understood three-dimensional space until we resolved the Poincaré conjecture. I personally encountered a similar situation in my efforts to solve the Calabi Conjecture: I believed that only after solving the Calabi Conjecture can we understand the concept of Ricci curvature in Kähler geometry.

Mathematicians should keep alive the discoveries of the past and enlighten the future. Here, in order to "keep alive the past" one must solve a challenging problem, while to "enlighten the future" one needs to develop deeper theoretical structures and search for new questions. Mathematics will die without new questions; thus to "enlighten the future" is the common imperative of all mathematicians. Our ultimate target is to integrate the natural sciences, the social sciences, and engineering-with mathematics as the foundation.

In the wake of Andrew Wiles' solution of Fermat's Last Theorem, many people wondered where the practical applications of such an achievement lie. Although all of us mathematicians regard the proof of Fermat's Last Theorem as epoch-making and therefore do not give a moment of thought to such concerns about applications, it is nevertheless worthwhile to confront this issue. It not only resolved a 350 year old puzzle, but it also allowed us to more deeply understand elliptic curves over rational number fields. Tremendous sparks burst forth from this combination of number theory/automorphic forms and elliptic curves. It is now worth mentioning that elliptic curves have flourished in coding theory, and consequently this understanding will be immensely useful in online trading. Its potential is beyond speculation.

Finally let us talk briefly about the difference between physicists and mathematicians. Generally, no permanent truth exists in the field of physics, in spite of physicists' grand efforts to conquer nature by persistently exploring the workings of the universe, and their hope to find the ultimate unified field theory. This statement is based on the fact that the entire truth of nature is too difficult for human beings to fully comprehend. In the realm of mathematics, however, every theorem can be strictly deduced from the axioms of the system, and are therefore irrefutable truths. As we have seen, mathematicians hold beauty as the main standard for selection. Good theorems allow us to feel and experience both the beauty and truth of nature, while leisurely reaching the state of "heaven, earth and I exist as a whole, and everything is with me." Our abstract view of nature is different from physicists' aspiration to understand nature.

Physicists make leaps in their thoughts in the hopes of capturing some truth of nature. Although this method is not rigorous, and one can easily make mistakes, it nevertheless allows physicists to arrive at a more thorough and more penetrating understanding of nature-a characteristic which is admired deeply by the mathematical community. By contrast, mathematicians must be very careful to proceed step by step, which requires them to spend time eliminating any possible errors that can arise. These two methods are, therefore, complementary to each other; neither can be omitted in our search for truth.

In traditional Chinese culture, we often talk about establishing moral standards without discussing the pursuit of truth, but how can we establish moral standards if we do not seek the truth? Confucius said that "to be gentle and magnanimous is the result of education by poetry." This is vaguely talking about beauty-how can we be gentle if we do not pursue beauty? Mathematics is the pursuit of both truth and beauty, and as such it is, without any doubt, the fundamental science most vital to China. 